\title{
ON PROLIFIC INDIVIDUALS IN A SUPERCRITICAL CONTINUOUS-STATE BRANCHING PROCESS
}

\author{
JEAN BERTOIN, ${ }^{*}$ Université Paris 6 and École Normale Supérieure \\ JOAQUIN FONTBONA ***** AND \\ SERVET MARTÍNEZ,****** Universidad de Chile
}

\begin{abstract}
We describe the genealogy of individuals with infinite descent in a supercritical continuous-state branching process.

Keywords: Continuous-state branching process; branching mechanism; extinction; reduced tree

2000 Mathematics Subject Classification: Primary 60J80

Secondary 60E10
\end{abstract}

\section{Introduction}

Consider a supercritical Galton-Watson process $Z$ with reproduction law $\pi$, where $\pi$ is a probability measure on $\mathbb{Z}_{+}$with $\sum_{n=0}^{\infty} n \pi(n) \in(1, \infty]$. We also assume that $\pi(0)>0$, and we write

$$
g(s):=\sum_{n=0}^{\infty} s^{n} \pi(n), \quad s \in[0,1],
$$

for the generating function of $\pi$. Then the following assertions are well known and easy to check; see, e.g. Proposition 11.8 of [9]. To start with, the equation $g(s)=s$ has a unique root $\rho$ in $(0,1)$, which coincides with the probability of extinction of $Z$ when the process starts from a single ancestor. Furthermore, splitting the graph of the generating function at $(\rho, \rho)$ produces the following pair of generating functions (see Figure 1):

$$
g_{e}(s):=\rho^{-1} g(\rho s)
$$

and

$$
g_{p}(s):=(1-\rho)^{-1} g(\rho+(1-\rho) s), \quad s \in[0,1] .
$$

More precisely, on the one hand, $g_{e}$ is the generating function of the subcritical reproduction law $\pi_{e}$ of the Galton-Watson process $Z_{e}$, which is obtained by conditioning $Z$ to become extinct, i.e.

$$
g_{e}(s)=\sum_{n=0}^{\infty} s^{n} \pi_{e}(n) \quad \text { with } \quad \pi_{e}(n):=\rho^{n-1} \pi(n) .
$$

Received 28 March 2007; revision received 17 June 2008.

* Postal address: Laboratoire de Probabilités et Modèles Aléatoires, Université Paris 6, 175 rue de Chevaleret, F-75013

Paris, France. Email address: jean.bertoin@upmc.fr

** Postal address: CMM-DIM, Universidad de Chile, Casilla 170-3, Correo 3, Santiago, Chile.

*** Email address: fontbona@dim.uchile.cl

**** Email address: smartine@dim.uchile.cl 


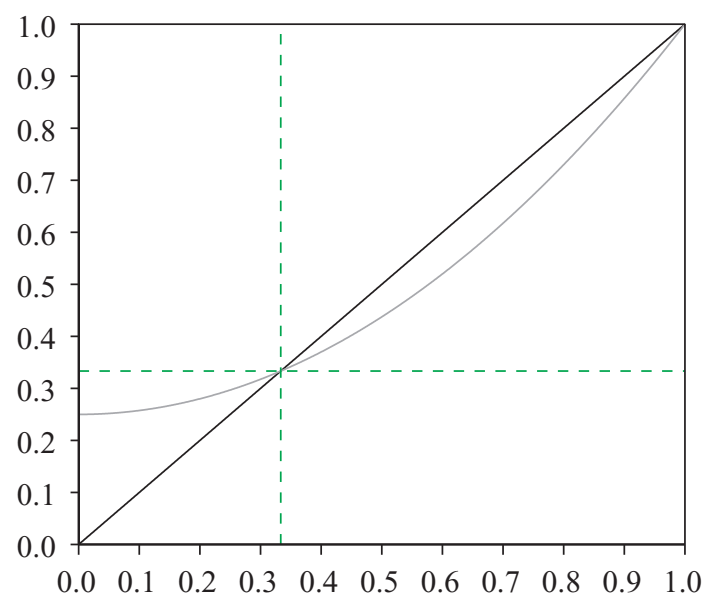

Figure 1: Graph of the generating function $g(s)=\frac{1}{4}+\frac{3}{4} s^{2}$ split at $\rho=\frac{1}{3}$; rescaling the lower-left quadrant and the upper-right quadrant yields the generating functions $g_{e}$ and $g_{p}$, respectively.

On the other hand, let us refer to an individual with infinite descent in the Galton-Watson process as a prolific individual. Then $g_{p}$ is the generating function of the reproduction law $\pi_{p}$ of the Galton-Watson process $Z_{p}$, which is obtained by the restriction of $Z$ to prolific individuals, i.e.

$$
g_{p}(s)=\sum_{\ell=1}^{\infty} s^{\ell} \pi_{p}(\ell) \quad \text { with } \quad \pi_{p}(\ell):=\sum_{n=\ell}^{\infty}\left(\begin{array}{l}
n \\
\ell
\end{array}\right)(1-\rho)^{\ell-1} \rho^{n-\ell} \pi(n) .
$$

In other words, the genealogical tree induced by $Z_{p}$ is distributed as that of $Z$ after conditioning on nonextinction and removing all the finite branches.

The purpose of this note is to point at an analog of these transformations in the framework of continuous-state branching processes (CSBPs). More precisely, the dynamics of a CSBP are characterized by a branching mechanism $\Psi$, which, in some loose sense, is related to the generating function $g$ of the reproduction law for Galton-Watson processes. It is well known that conditioning a supercritical CSBP to become (eventually) extinguished yields another CSBP whose branching mechanism $\Psi_{e}$ is a simple transformation of $\Psi$. Our main interest here is to show that the notion of prolific individuals can also be defined for a CSBP and yields a continuous-time (but discrete space) branching process whose characteristics are again expressed by simple transforms of the original CSBP. It will certainly not come as a surprise that a result for Galton-Watson processes possesses a counterpart in the continuous setting, and indeed, a closely related description has been obtained recently by Duquesne and Winkel [4]. Our approach differs from that of Duquesne and Winkel and enables us to handle more general CSBPs. Specifically, we will essentially rely on the connection between CSBPs and subordinators, whereas Duquesne and Winkel dwelled on techniques from real trees and percolation on Galton-Watson trees. Finally, in the case of stable branching mechanisms, this points at some simple path transformations which do not seem to have been observed previously.

We refer the reader to [6, Chapter 10], [8], and the references therein for background on CSBPs. We begin by recalling the material that will be needed here. 


\section{Preliminaries}

Consider a conservative CSBP $X=(X(t, a): t \geq 0$ and $a \geq 0)$, where $t$ is the time parameter and $a$ is the size of the initial population. This means that, for each fixed $a \geq 0$, the process $X(\cdot, a)$ is a time-homogeneous Markov process with values in $\mathbb{R}_{+}$started from $X(0, a)=a$. Furthermore, the fundamental branching property holds, namely, for every $a, b \geq 0, X(\cdot, a+b)-X(\cdot, a)$ has same the law as $X(\cdot, b)$ and is independent of the family of processes $(X(\cdot, c), 0 \leq c \leq a)$.

The dynamics of $X$ are characterized by its branching mechanism $\Psi:[0, \infty) \rightarrow \mathbb{R}$, which is a convex function of the type

$$
\Psi(q)=\alpha q+\beta q^{2}+\int_{(0, \infty)}\left(\mathrm{e}^{-q x}-1+q x \mathbf{1}_{\{x \leq 1\}}\right) \Pi(\mathrm{d} x),
$$

where $\alpha \in \mathbb{R}, \beta \geq 0$, and $\Pi$ is a measure on $(0, \infty)$ such that $\int\left(1 \wedge x^{2}\right) \Pi(\mathrm{d} x)<\infty$. Specifically, the semigroup of $X(\cdot, a)$ can be characterized via its Laplace transform as follows. For every $q>0$, we have

$$
\mathrm{E}(\exp \{-q X(t, a)\})=\exp \left\{-a u_{t}(q)\right\}
$$

where the function $u_{t}(q)$ solves

$$
\frac{\partial u_{t}(q)}{\partial t}=-\Psi\left(u_{t}(q)\right), \quad u_{0}(q)=q
$$

We will assume throughout this work that $X$ is supercritical, i.e. that

$$
\Psi^{\prime}(0+)=\alpha-\int_{(1, \infty)} x \Pi(\mathrm{d} x) \in[-\infty, 0),
$$

and not immortal, in the sense that $\Psi(q)>0$ when $q$ is sufficiently large. As the branching mechanism is a convex function with $\Psi(0)=0$, this implies that there exists a unique $q_{0}>0$ that solves the equation

$$
\Psi\left(q_{0}\right)=0 .
$$

We also recall that the hypothesis that $X$ is conservative (i.e. the process $X(\cdot, a)$ does not explode in finite time almost surely) is then equivalent to $\int_{0+}|\Psi(q)|^{-1} \mathrm{~d} q=\infty$ (see [5]).

The importance of the role of the positive root $q_{0}$ of the branching mechanism should already be clear from the following easy consequence of (1) and (2). For each $a \geq 0$, the process $\exp \left\{-q_{0} X(\cdot, a)\right\}$ is a martingale with values in $[0,1]$; it thus converges almost surely and it is easily seen that its limit can only take the values 0 or 1 almost surely. More precisely, writing $X(\infty, a)=\lim _{t \rightarrow \infty} X(t, a)$, we have

$$
\mathrm{P}(X(\infty, a)=0)=1-\mathrm{P}(X(\infty, a)=\infty)=\exp \left\{-q_{0} a\right\} \quad \text { for all } a \geq 0 .
$$

In the sequel we will say that the CSBP with initial population of size $a$ becomes eventually extinguished when $X(\infty, a)=0$ and is prolific when $X(\infty, a)=\infty$. We mention that the process may become eventually extinguished without ever being entirely extinguished: the event that $X(\infty, a)=0$ and $X(t, a)>0$ for all $t \geq 0$ has a positive probability whenever the branching mechanism fulfills $\int^{\infty} \Psi^{-1}(q) \mathrm{d} q=\infty$. 
In order to rigorously define prolific individuals, we turn our attention to specifying the genealogy in a CSBP, which requires the connection with subordinators and Bochner subordination. Specifically, the branching property entails that, for each fixed $t \geq 0$, the process $X(t, \cdot)$ has independent and homogeneous increments with values in $\mathbb{R}_{+}$. We will always deal with its right-continuous modification which is then a subordinator. We see from (1) that its Laplace exponent is the function $q \rightarrow u_{t}(q)$, and the semigroup identity $u_{t+s}(q)=u_{t}\left(u_{s}(q)\right)$ points at the following representation (see Proposition 1 of [2] for details).

Lemma 1. On some probability space, there exists a process $\left(S^{(s, t)}(a), 0 \leq s \leq t\right.$ and $\left.a \geq 0\right)$ such that the following statements hold.

(i) For every $0 \leq s \leq t, S^{(s, t)}=\left(S^{(s, t)}(a), a \geq 0\right)$ is a subordinator with Laplace exponent $u_{t-s}(\cdot)$.

(ii) For every integer $p \geq 2$ and $0 \leq t_{1} \leq \cdots \leq t_{p}$, the subordinators $S^{\left(t_{1}, t_{2}\right)}, \ldots, S^{\left(t_{p-1}, t_{p}\right)}$ are independent and

$$
S^{\left(t_{1}, t_{p}\right)}(a)=S^{\left(t_{p-1}, t_{p}\right)} \circ \cdots \circ S^{\left(t_{1}, t_{2}\right)}(a) \text { for all } a \geq 0 \text { almost surely. }
$$

(iii) The processes $\left(S^{(0, t)}(a), t \geq 0\right.$ and $\left.a \geq 0\right)$ and $(X(t, a), t \geq 0$ and $a \geq 0)$ have the same finite-dimensional marginals.

For the sake of simplicity, from now on we will further assume that

$$
\beta>0 \quad \text { or } \int_{(0, \infty)}(1 \wedge x) \Pi(\mathrm{d} x)=\infty,
$$

in order to ensure that the subordinators $S^{(s, t)}$ are pure-jump processes (i.e. they have no drift); see [12]. Analyzing their jumps in the framework of the representation above yields a natural notion of genealogy of CSBPs (we refer the reader to [2] for details): for every $b, c \geq 0$ and $0 \leq s<t$, we say that individual $c$ in the population at time $t$ has ancestor (or is a descendant of) individual $b$ in the population at time $s$ if $b$ is a jump time of $S^{(s, t)}$ and

$$
S^{(s, t)}(b-)<c<S^{(s, t)}(b) .
$$

Note that, when the subordinator $S^{(s, t)}$ has a jump at location $b$, the size of this jump $\Delta S^{(s, t)}(b)=$ $S^{(s, t)}(b)-S^{(s, t)}(b-)$ describes the size of the subpopulation at time $t$ which descends from individual $b$ in the population at time $s$. Considering the limit as $t \rightarrow \infty$, this enables us to define prolific individuals.

Definition. For every $b \geq 0$ and $s \geq 0$, we say that individual $b$ in the population at time $s$ is prolific if

$$
\lim _{t \rightarrow \infty} \Delta S^{(s, t)}(b)=\infty .
$$

For every $a \geq 0$ and $s \geq 0$, we introduce the number of prolific individuals in the population at time $s$ which descend from the initial population $[0, a]$ of size $a$,

$$
P(s, a):=\operatorname{card}\{b \in[0, X(s, a)]: b \text { is a prolific individual in the population at time } s\} .
$$

We point out that there are prolific individuals in the initial population if and only if the CSBP is prolific. This is certainly not surprising, but it requires a rigorous argument which has some importance in this study. 
Lemma 2. For every initial population $a \geq 0$, the events

$$
\{X(\cdot, a) \text { becomes eventually extinguished }\}
$$

and

$$
\{P(0, a)=0\}
$$

coincide almost surely. Furthermore, $P(0, a)$ has the Poisson distribution with parameter $a q_{0}$.

Proof. As the inclusion

$$
\{X(\cdot, a) \text { becomes eventually extinguished }\} \subseteq\{P(0, a)=0\}
$$

is obvious, we just need to check that the probability of the two events coincide.

Fix an arbitrary time $t>0$ and focus on the evolution of the initial population $[0, a]$. The fact that the subordinator $S^{(0, t)}$ is pure jump means that almost all the population at time $t$ descends from at most countably many individuals in the initial population. More precisely, denote by $\left(a_{i}\right)_{i \in I}$ the set of jump locations of $S^{(0, t)}(\cdot)$ on $[0, a]$; so $\Delta S^{(0, t)}\left(a_{i}\right)$ is the size of the subpopulation at time $t$ having $a_{i}$ as ancestor and

$$
\sum_{i \in I} \Delta S^{(0, t)}\left(a_{i}\right)=X(t, a)
$$

Since, for every $t^{\prime}>t$, the pure-jump subordinator $S^{\left(t, t^{\prime}\right)}$ is independent of $S^{(0, t)}$ and $S^{\left(0, t^{\prime}\right)}=S^{\left(t, t^{\prime}\right)} \circ S^{(0, t)}$, we see that the ancestors in the population at time $t$ of the almost entire population at time $t^{\prime}$ descend from the individuals $\left(a_{i}\right)_{i \in I}$. As a consequence, any prolific individual in the initial population belongs to the set of ancestors $\left(a_{i}\right)_{i \in I}$. By applying the branching property at time $t$, we find that the conditional probability, given the evolution of the process up to time $t$ that the individual $a_{i}$ is prolific, equals $1-\exp \left\{-q_{0} \Delta S^{(0, t)}\left(a_{i}\right)\right\}$, and, for different indices $i$, these events are (conditionally) independent. Thus,

$$
\begin{aligned}
\mathrm{P}(P(0, a)=0) & =\mathrm{E}\left(\prod_{i \in I} \exp \left\{-q_{0} \Delta S^{(0, t)}\left(a_{i}\right)\right\}\right) \\
& =\mathrm{E}\left(\exp \left\{-q_{0} \sum_{i \in I} \Delta S^{(0, t)}\left(a_{i}\right)\right\}\right) \\
& =\mathrm{E}\left(\exp \left\{-q_{0} X(t, a)\right\}\right) \\
& =\exp \left\{-q_{0} a\right\} \\
& =\mathrm{P}(X(\cdot, a) \text { becomes eventually extinguished }) .
\end{aligned}
$$

This shows the first assertion. Finally, the branching property entails that the process $P(0, \cdot)$ is Poisson, and since $\mathrm{P}(P(0, a)=0)=\exp \left\{-q_{0} a\right\}$, its intensity is $q_{0}$.

Remark 1. An application of the Markov property shows that, conditionally on $X(t, a)=b$, the number of prolific individuals at time $t$ has the Poisson law with parameter $q_{0} b$. By the law of large number for the Poisson laws, we deduce that, conditionally on the event that $X(\cdot, a)$ is prolific, we have $P(t, a) \sim q_{0} X(t, a)$ as $t \rightarrow \infty$. 


\section{Main results}

Fix $a>0$, and introduce the probability measure

$$
\mathrm{P}_{e}=\exp \left\{q_{0} a\right\} \mathbf{1}_{\{X(a, \infty)=0\}} \mathrm{P},
$$

which is obtained by conditioning the CSBP with initial population of size $a$ to become eventually extinguished. Observe that, on the $\sigma$-field $\mathcal{F}_{t}=\sigma(X(r, a): 0 \leq r \leq t), \mathrm{P}_{e}$ is absolutely continuous with respect to the initial probability measure $\mathrm{P}$ with density given by the martingale $\exp \left\{q_{0} a\right\} \exp \left\{-q_{0} X(t, a)\right\}$.

We now have all the ingredients needed to state and prove the main results of this note. First, let us present the continuous-time analogue of the interpretation of the component $g_{e}$ for Galton-Watson processes, which belongs to the folklore of CSBPs.

Proposition 1. Under $\mathrm{P}_{e}, X(\cdot, a)$ is a CSBP with initial population of size a. Its branching mechanism is given by

$$
\Psi_{e}(q)=\Psi\left(q_{0}+q\right), \quad q \geq 0,
$$

and can be expressed in the form

$$
\Psi_{e}(q)=\alpha_{e} q+\beta q^{2}+\int_{(0, \infty)}\left(\mathrm{e}^{-q x}-1+q x \mathbf{1}_{\{x \leq 1\}}\right) \Pi_{e}(\mathrm{~d} x),
$$

where

$$
\Pi_{e}(\mathrm{~d} x)=\exp \left\{-q_{0} x\right\} \Pi(\mathrm{d} x)
$$

and

$$
\alpha_{e}=\alpha+2 \beta q_{0}+\int_{(0, \infty)}\left(1-\exp \left\{-q_{0} x\right\}\right) x \mathbf{1}_{\{x \leq 1\}} \Pi(\mathrm{d} x) .
$$

More generally, we point out how a simple modification of the law $\mathrm{P}_{e}$ of the branching process $X(\cdot, a)$ conditioned to become eventually extinguished enables us to describe the conditional distribution of $X(t, a)$ on the number of prolific individuals $P(t, a)$ at a fixed time $t>0$.

Proposition 2. For every $a, t \geq 0$ and $n \in \mathbb{Z}_{+}$, the conditional law of $X(t, a)$ given $P(t, a)=$ $n$ is

$$
\mathrm{P}(X(t, a) \in \mathrm{d} x \mid P(t, a)=n)=x^{n} \frac{\mathrm{P}_{e}(X(t, a) \in \mathrm{d} x)}{\mathrm{E}_{e}\left(X(t, a)^{n}\right)} .
$$

Proof. To start with, Remark 1 yields the identity

$$
\begin{aligned}
\mathrm{E}\left(\exp \{-q X(t, a)\} s^{P(t, a)}\right) & =\mathrm{E}\left(\exp \{-q X(t, a)\} \exp \left\{-q_{0}(1-s) X(t, a)\right\}\right) \\
& =\mathrm{E}\left(\exp \left\{-\left(q+q_{0}\right) X(t, a)\right\} \exp \left\{q_{0} s X(t, a)\right\}\right) \\
& =\sum_{n=0}^{\infty} \frac{\left(s q_{0}\right)^{n}}{n !} \mathrm{E}\left(\exp \left\{-\left(q+q_{0}\right) X(t, a)\right\} X(t, a)^{n}\right) .
\end{aligned}
$$

Next, we define

$$
f(t, q, a, n):=\mathrm{E}(\exp \{-q X(t, a)\} \mid P(t, a)=n) .
$$

Again using Remark 1, but conditioning first on $P(t, a)$ and then on $X(t, a)$, we obtain

$$
\mathrm{E}\left(\exp \{-q X(t, a)\} s^{P(t, a)}\right)=\sum_{n=0}^{\infty} \frac{\left(s q_{0}\right)^{n}}{n !} f(t, q, a, n) \mathrm{E}\left(\exp \left\{-q_{0} X(t, a)\right\} X(t, a)^{n}\right) .
$$


We deduce that

$$
\begin{aligned}
f(t, q, a, n) & =\frac{\mathrm{E}\left(\exp \left\{-\left(q+q_{0}\right) X(t, a)\right\} X(t, a)^{n}\right)}{\mathrm{E}\left(\exp \left\{-q_{0} X(t, a)\right\} X(t, a)^{n}\right)} \\
& =\frac{\mathrm{E}_{e}\left(\exp \{-q x(t, a)\} X(t, a)^{n}\right)}{\mathrm{E}_{e}\left(X(t, a)^{n}\right)},
\end{aligned}
$$

where in the second identity we made use of the fact that, on the $\sigma$-field $\mathcal{F}_{t}=\sigma(X(r, a): 0 \leq$ $r \leq t)$, the probability measure $\mathrm{P}_{e}$ for the CSBP conditioned to become eventually extinguished is absolutely continuous with respect to the initial probability measure $\mathrm{P}$ with density $\exp \left\{q_{0} a\right\} \exp \left\{-q_{0} X(t, a)\right\}$. Inverting the Laplace transform (in $q$ ) yields the formula of the statement.

Recall that, with any probability law $m$ on $\mathbb{R}_{+}$with finite nonzero mean, we can associate the law $\bar{m}$ of its size-biased picking, defined by

$$
\bar{m}(\mathrm{~d} y)=\frac{y}{c} m(\mathrm{~d} y)
$$

with $c=\int_{0}^{\infty} y m(\mathrm{~d} y)$. We may then note the following recursive identity: for every $n \in \mathbb{Z}_{+}$, the law $\mathcal{L}(X(t, a) \mid P(t, a)=n+1)$ is obtained from $\mathcal{L}(X(t, a) \mid P(t, a)=n)$ by size-biased picking.

In order to state the main result of this note, we first recall some further well-known material (see, e.g. Chapter III of [1] or [7]). A continuous-time branching process $Z=(Z(t, k): t \geq$ $0, k \in \mathbb{Z}_{+}$), where $t$ is the time parameter and $k$ is the number of ancestors, can be viewed as a Galton-Watson process in which individuals have independent, exponentially distributed lifetimes. The rate of reproduction is governed by a finite measure $\mu$ on $\mathbb{Z}_{+}$with $\mu(1)=0$, which we call the reproduction measure. Specifically, each individual lives for an exponential time with parameter $\mu\left(\mathbb{Z}_{+}\right)$and begets at its death a random number of children that is distributed according to the normalized probability measure $\mu(\cdot) / \mu\left(\mathbb{Z}_{+}\right)$(which coincides with the reproduction law of the underlying Galton-Watson process). Thus, for each $k \in \mathbb{Z}_{+}$, $Z(\cdot, k)$ is a Markov chain in continuous time, whose dynamics are entirely characterized by the reproduction measure $\mu$. In turn, the latter is determined by the function

$$
\Phi(s):=\sum_{n=0}^{\infty}\left(s^{n}-s\right) \mu(n), \quad s \in[0,1] .
$$

More precisely, the branching property entails that the generating function of $Z(t, k)$ has the form

$$
\mathrm{E}\left(s^{Z(t, k)}\right)=\gamma_{t}(s)^{k}, \quad s \in[0,1], k \in \mathbb{Z}_{+},
$$

and solves

$$
\frac{\partial \gamma_{t}(s)}{\partial t}=\Phi\left(\gamma_{t}(s)\right)
$$

In the case when $\mu(0)=0$, we say that $Z$ is immortal as each individual has at least two children almost surely.

Theorem 1. For every $a \geq 0$, the process $P(\cdot, a)$ is an immortal branching process in continuous time with initial distribution given by the Poisson law with parameter $q_{0} a$. Its reproduction measure $\mu_{p}$ is characterized in terms of the branching mechanism of $X$ by

$$
\Phi_{p}(s)=\sum_{n=0}^{\infty}\left(s^{n}-s\right) \mu_{p}(n)=\frac{1}{q_{0}} \Psi\left(q_{0}(1-s)\right), \quad s \in[0,1],
$$




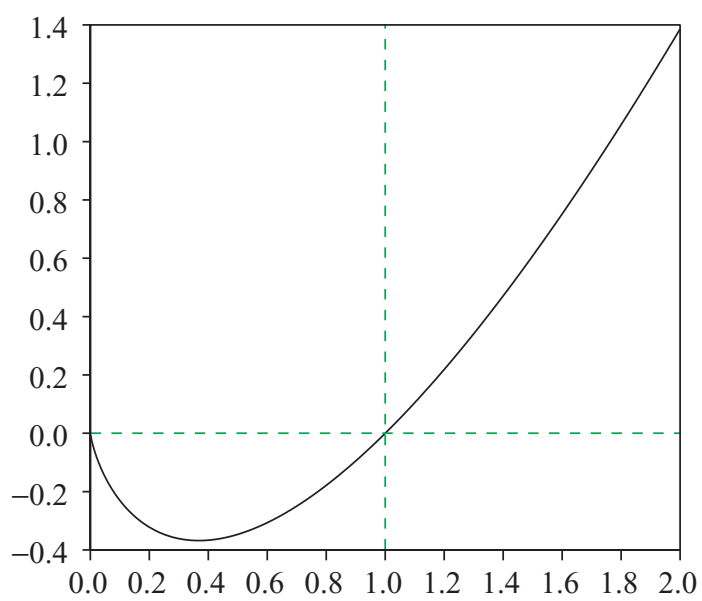

FIGURE 2: Graph of the branching mechanism $\Psi(q)=q \ln q$ split at $q_{0}=1$. The upper-right quadrant gives the graph of $\Psi_{e}$ and the reflection of the lower-left quadrant gives the graph of $\Phi_{p}$.

and is explicitly given by

$$
\mu_{p}(n)=q_{0}^{n-1} \int_{(0, \infty)} \frac{x^{n}}{n !} \exp \left\{-q_{0} x\right\} \Pi(\mathrm{d} x) \text { for } n \geq 3
$$

and

$$
\mu_{p}(2)=\beta q_{0}+q_{0} \int_{(0, \infty)} \frac{x^{2}}{2} \exp \left\{-q_{0} x\right\} \Pi(\mathrm{d} x) .
$$

Figure 2 depicts the transformation $\Psi \rightarrow\left(\Psi_{e}, \Phi_{p}\right)$ and should be compared to Figure 1 for generating functions in the setting of Galton-Watson processes.

Proof of Theorem 1. The proof of the assertion that the process $P(\cdot, a)$ of the number of prolific individuals is a branching process in continuous time follows the same route as GaltonWatson processes by using the argument in the proof of Lemma 2. That this branching process is immortal is obvious. Lemma 2 also states that its initial distribution is the Poisson law with parameter $a q_{0}$.

Let us now compute the generating function of its semigroup. Recall that the generating function of the Poisson distribution with parameter $c \geq 0$ is $s \rightarrow \exp \{-(1-s) c\}$. On the one hand, combining Lemma 2 with the Markov property at time $t$ yields

$$
\begin{aligned}
\mathrm{E}\left(s^{P(t, a)}\right) & =\mathrm{E}\left(\exp \left\{-(1-s) q_{0} X(t, a)\right\}\right) \\
& =\exp \left\{-a u_{t}\left((1-s) q_{0}\right)\right\} .
\end{aligned}
$$

On the other hand, using the fact that $P(0, a)$ has the Poisson distribution with parameter $a q_{0}$ shows that the generating function $\gamma_{t}$ of the continuous-time branching process fulfills

$$
\mathrm{E}\left(s^{P(t, a)}\right)=\mathrm{E}\left(\gamma_{t}(s)^{P(0, a)}\right)=\exp \left\{-a q_{0}\left(1-\gamma_{t}(s)\right)\right\} .
$$

We deduce from these two observations that

$$
1-\gamma_{t}(s)=\frac{1}{q_{0}} u_{t}\left((1-s) q_{0}\right) .
$$


Taking the derivative in $t$ yields, by (2) and (3),

$$
\Phi_{p}\left(\gamma_{t}(s)\right)=\frac{1}{q_{0}} \Psi\left(u_{t}\left((1-s) q_{0}\right)\right)=\frac{1}{q_{0}} \Psi\left(q_{0}\left(1-\gamma_{t}(s)\right)\right) .
$$

We conclude that

$$
\Phi_{p}(s)=\frac{1}{q_{0}} \Psi\left(q_{0}(1-s)\right) .
$$

Finally, we recover the measure $\mu_{p}$ by inverting of the transform $\Phi_{p}$. This can be performed by combining Proposition 1 and the observation that $\Psi\left(q_{0}(1-s)\right)=\Psi_{e}\left(-q_{0} s\right)$.

Remark. When the branching mechanism fulfills the additional condition

$$
\int^{\infty} \Psi^{-1}(q) \mathrm{d} q<\infty
$$

(this requirement is equivalent to the assumption that asymptotic extinction coincides with extinction in finite time, which is needed to ensure the separability of the associated real tree in [4]), we can use the link between CSBPs and Lévy trees, and identify branches with infinite length in a continuous tree as prolific individuals. Then we can view Theorem 1 as a special case of Theorem 5.6 of [4], which characterizes the law of the infinite component in a Lévy tree as a Galton-Watson tree with exponential edge lengths.

In the setting of supercritical Galton-Watson processes we can assign a type to each individual depending on whether it has finite descent or is prolific, and this yields two-type GaltonWatson processes. A similar observation can be made in the continuous setting; in this direction recall that, for every fixed $t \geq 0$, there are only countably many prolific individuals at time $t$, which thus do not contribute to the size of the population at time $t$ (but of course the descent of prolific individuals at time $t$ may have a crucial role in the size of the population at time $t^{\prime}>t$ ). Then we can check that the pair $((X(t, a), P(t, a)): a, t \geq 0)$ also satisfies the branching property. More precisely, for every $a \geq 0$ and $n \in \mathbb{Z}_{+}$, let us write $(X(\cdot, a, n), P(\cdot, a, n))$ for a version of the pair of processes $(X(t, a), P(t, a))_{t \geq 0}$ conditioned on $P(0, a)=n$. Then, for every $a, a^{\prime} \geq 0$ and $n, n^{\prime} \in \mathbb{Z}_{+}$, there is the identity in distribution

$$
\begin{aligned}
& \left(X\left(\cdot, a+a^{\prime}, n+n^{\prime}\right), P\left(\cdot, a+a^{\prime}, n+n^{\prime}\right)\right) \\
& \quad \stackrel{\mathcal{L}}{=}(X(\cdot, a, n), P(\cdot, a, n))+\left(X^{\prime}\left(\cdot, a^{\prime}, n^{\prime}\right), P^{\prime}\left(\cdot, a^{\prime}, n^{\prime}\right)\right),
\end{aligned}
$$

where, on the right-hand side, $\left(X^{\prime}\left(\cdot, a^{\prime}, n^{\prime}\right), P^{\prime}\left(\cdot, a^{\prime}, n^{\prime}\right)\right)$ is independent of $(X(\cdot, a, n)$, $P(\cdot, a, n))$ and has the same law as $\left(X\left(\cdot, a^{\prime}, n^{\prime}\right), P\left(\cdot, a^{\prime}, n^{\prime}\right)\right)$.

For $n=0, X(\cdot, a, 0)$ is just a version of the initial CSBP with an initial population of size $a$ and conditioned to become eventually extinguished (i.e. with branching mechanism $\left.\Psi_{e}(q)=\Psi\left(q+q_{0}\right)\right)$, and, obviously, $P(\cdot, a, 0) \equiv 0$. This yields

$$
\mathrm{E}\left(\exp \{-q X(t, a, 0)\} s^{P(t, a, 0)}\right)=\exp \left\{-a\left(u_{t}\left(q+q_{0}\right)-q_{0}\right)\right\}
$$

for all $q \geq 0$ and $s \in(0,1]$, where $u_{t}(\cdot)$ is defined as in (2). Next, recall from Remark 1 that there is the identity

$$
\begin{aligned}
\mathrm{E}\left(\exp \{-q X(t, a)\} s^{P(t, a)}\right) & =\mathrm{E}\left(\exp \left\{-\left(q+q_{0}(1-s)\right) X(t, a)\right\}\right) \\
& =\exp \left\{-a u_{t}\left(q+q_{0}(1-s)\right)\right\} .
\end{aligned}
$$


On the other hand, since $P(0, a)$ has the Poisson distribution with parameter $a q_{0}$, the branching property enables us to express the preceding quantity as

$$
\begin{aligned}
& \mathrm{E}\left(\exp \{-q X(t, a)\} s^{P(t, a)}\right) \\
& \quad=\sum_{n=0}^{\infty} \exp \left\{-a q_{0}\right\} \frac{\left(a q_{0}\right)^{n}}{n !} \mathrm{E}\left(\exp \{-q X(t, a, 0)\} s^{P(t, a, 0)}\right)\left(\mathrm{E}\left(\exp \{-q X(t, 0,1)\} s^{P(t, 0,1)}\right)\right)^{n} .
\end{aligned}
$$

Using (5) and considering the asymptotic when $a \rightarrow 0$, easily yield

$$
\mathrm{E}\left(\exp \{-q X(t, 0,1)\} s^{P(t, 0,1)}\right)=\frac{1}{q_{0}}\left(u_{t}\left(q+q_{0}\right)-u_{t}\left(q+q_{0}(1-s)\right)\right) .
$$

Putting the pieces together, we conclude that the joint law of $(X(t, a, n), P(t, a, n))$ is characterized by

$$
\begin{aligned}
\mathrm{E}\left(\exp \{-q x(t, a, n)\} s^{P(t, a, n)}\right)= & \exp \left\{-a\left(u_{t}\left(q+q_{0}\right)-q_{0}\right)\right\} \\
& \times\left(\frac{1}{q_{0}}\left(u_{t}\left(q+q_{0}\right)-u_{t}\left(q+q_{0}(1-s)\right)\right)\right)^{n} .
\end{aligned}
$$

\section{Some examples}

We will now present some examples in which explicit computations are possible. The third example will point at a path transformation that relates strictly stable CSBPs to some supercritical CSBPs which may be new.

Example 1. (Quadratic branching.) The simplest example is when $\Psi(q)=q^{2}-q$, so $\Pi=0$, $\beta=1$, and $\alpha=-1$. Then we obtain $q_{0}=1$ and $\Phi_{p}(s)=s^{2}-s$, which yield $\mu_{p}=\delta_{2}$. We conclude that, as time passes, the number of prolific individuals evolves as a standard Yule process. This is a well-known fact; see, for instance, [11].

Example 2. (Neveu's branching.) Next, we consider Neveu's branching process [2], [10], which has branching mechanism $\Psi(q)=q \ln (q)$. Then $q_{0}=1$ and

$$
\Phi_{p}(s)=(1-s) \ln (1-s)=\sum_{n=2}^{\infty} \frac{s^{n}-s}{n(n-1)} .
$$

We thus obtain $\mu_{p}(n)=1 / n(n-1)$ for every $n \geq 2$. As a check, recall that Neveu's branching process has no Gaussian component and that its Lévy measure is $\Pi(\mathrm{d} x)=x^{-2} \mathrm{~d} x$, and, thus, we recover from (4) that, for $n \geq 2$,

$$
\mu_{p}(n)=\int_{0}^{\infty} \frac{x^{n}}{n !} \mathrm{e}^{-x} x^{-2} \mathrm{~d} x=\frac{(n-2) !}{n !}=\frac{1}{n(n-1)} .
$$

We also point out that $u_{t}(q)=q^{\mathrm{e}^{-t}}$, and, thus,

$$
\gamma_{t}(s)=1-(1-s)^{\mathrm{e}^{-t}}, \quad s \in[0,1]
$$

Example 3. (Stable branching.) Next consider the supercritical stable branching mechanism $\Psi(q)=\Gamma(-\vartheta)\left(q^{\vartheta}-q\right)$, so that $q_{0}=1$ and $\Pi(\mathrm{d} x)=x^{-\vartheta-1} \mathrm{~d} x$, and

$$
\mu_{p}(n)=\int_{0}^{\infty} \frac{x^{n}}{n !} \mathrm{e}^{-x} x^{-\vartheta-1} \mathrm{~d} x=\frac{\Gamma(n-\vartheta)}{n !}, \quad n \geq 2 .
$$


It is easily checked that the total mass of $\mu_{p}$ is

$$
\mu_{p}\left(\mathbb{Z}_{+}\right)=\frac{\Gamma(2-\vartheta)}{\vartheta}=(\vartheta-1) \Gamma(-\vartheta)
$$

so the normalized probability measure $\mu_{p}(\cdot) / \mu_{p}\left(\mathbb{Z}_{+}\right)$is given by

$$
\frac{\mu_{p}(n)}{\mu_{p}\left(\mathbb{Z}_{+}\right)}=\frac{\vartheta(2-\vartheta) \cdots(n-1-\vartheta)}{n !}:=v_{\vartheta}(n), \quad n \geq 2 .
$$

The reproduction law $\left(v_{\vartheta}(n)\right)_{n \geq 2}$ in Example 3 already appeared at the bottom of page 74 of Duquesne and Le Gall [3] (see also Section 7 of [7]), which points at a rather surprising connection with strictly stable trees reduced at some finite level. More precisely, Duquesne and Le Gall (see Sections 2.6 and 2.7 of [3]) were interested in the limit of certain reduced critical Galton-Watson trees observed up to some large generation. Following Theorem 2.7.1 of [3], we consider a time-inhomogeneous Markov process $\left(Z_{t}^{1}\right)_{0 \leq t<1}$ with values in $\mathbb{N}$, which models the evolution of a population with the following dynamics. The death time of an individual that is alive at time $t \in[0,1)$ has the uniform distribution on $[t, 1]$, and at its death, this individual begets a random number of children distributed according to the reproduction law $v_{\vartheta}$, independently of the death time. Furthermore, different individuals evolve independently of the others. Heuristically, the quantity $Z_{t}^{1}$ can be interpreted as the number of individuals at time $t$ which have a nonzero descent at time 1 in a strictly stable( $\vartheta)$-CSBP, i.e. with branching mechanism $\Psi_{\vartheta}(q):=c q^{\vartheta}$, where $c>0$ is arbitrary.

On the other hand, recall that a random variable $\varepsilon$ which has the exponential distribution with parameter $c>0$ satisfies the property of absence of memory, and, furthermore, $1-\exp \{-c \varepsilon\}$ is then uniformly distributed on $[0,1]$. Putting these observations together, we now realize that if $Z^{1}$ starts with a number of ancestors distributed according to the Poisson law with parameter $a$ then the time-changed process

$$
t \rightarrow Z_{1-\exp \{-(\vartheta-1) \Gamma(-\vartheta) t\}}^{1}
$$

is a version of the process $(P(t, a))_{t \geq 0}$ of the number of prolific individuals for a CSBP with branching mechanism $\Psi(q)=\Gamma(-\vartheta)\left(q^{\vartheta}-q\right)$ and started from an initial population of size $a$.

We now conclude this work by providing a direct explanation for the preceding relation, which is based on the following simple transformation of strictly stable CSBPs.

Proposition 3. Let $(Y(t, a): t \geq 0$ and $a \geq 0)$ be a strictly stable CSBP with branching mechanism $\Psi_{\vartheta}(q)=c q^{\vartheta}$, where $c>0$ and $\bar{\vartheta} \in(1,2]$, and fix $b>0$. Then the process

$$
\tilde{Y}(t, a):=\mathrm{e}^{b t} Y\left(1-\mathrm{e}^{-b(1-\vartheta) t}, a\right), \quad t \geq 0 \text { and } a \geq 0,
$$

is a CSBP with branching mechanism

$$
\widetilde{\Psi}_{\vartheta}(q)=b c(\vartheta-1) q^{\vartheta}-b q .
$$

This provides a pathwise proof for the identity in distribution which was observed above. Indeed, we choose $b=\Gamma(-\vartheta)$ and $c=1 /(\vartheta-1)$ so that $\widetilde{\Psi}_{\vartheta}(q)=\Gamma(-\vartheta)\left(q^{\vartheta}-q\right)$. Then it suffices to observe that $Z_{1-\exp \{-(\vartheta-1) \Gamma(-\vartheta) t\}}^{1}$, the number of individuals at time $1-\mathrm{e}^{-b(1-\vartheta) t}$ which have a nonzero descent at time 1 in the strictly stable CSBP $Y$, coincides with the number 
of prolific individuals at time $t$ in the supercritical CSBP $\tilde{Y}$. For this, we have to use the feature that, since $\int^{\infty} \mathrm{d} q / \widetilde{\Psi}_{\vartheta}(q)<\infty$, the following equivalence holds with probability 1:

$$
\tilde{Y}(t, a)=0 \text { when } t \text { is sufficiently large } \Longleftrightarrow \quad \lim _{t \rightarrow \infty} \tilde{Y}(t, a)=0 .
$$

In other words, when the CSBP $\tilde{Y}$ becomes eventually extinguished, it must become entirely extinguished at some finite time. See, for instance, the exercise of [8, p. 28].

Proof of Proposition 3. Let us write

$$
v_{t}(q)=-\ln \mathrm{E}(\exp \{-q Y(t, 1)\})
$$

for the solution to (2) for the branching mechanism $\Psi_{\vartheta}(q)=c q^{\vartheta}$. This equation can be solved explicitly and we find that

$$
v_{t}(q)=\left((\vartheta-1) c t+q^{1-\vartheta}\right)^{1 /(1-\vartheta)}, \quad q>0 .
$$

It is immediate to check that the transformed process $\tilde{Y}$ is a (possibly time-inhomogeneous) Markov process that satisfies the branching property. Identity (1) yields

$$
\mathrm{E}(\exp \{-q \tilde{Y}(t, a)\})=\mathrm{E}\left(\exp \left\{-q \mathrm{e}^{b t} Y\left(1-\mathrm{e}^{-b(1-\vartheta) t}, a\right)\right\}\right)=\exp \left\{-a u_{t}(q)\right\}
$$

with

$$
\begin{aligned}
& u_{t}(q)=v_{1-\mathrm{e}^{-b(\vartheta-1) t}}\left(q \mathrm{e}^{b t}\right) \\
& =\left((\vartheta-1) c\left(1-\mathrm{e}^{-b(\vartheta-1) t}\right)+q^{1-\vartheta} \mathrm{e}^{-b(\vartheta-1) t}\right)^{1 /(1-\vartheta)} .
\end{aligned}
$$

Taking the derivative with respect to $t$, we obtain

$$
\begin{aligned}
\frac{\partial u_{t}(q)}{\partial t} & =\frac{1}{1-\vartheta}\left(b(\vartheta-1)^{2} c \mathrm{e}^{-b(\vartheta-1) t}-b(\vartheta-1) q^{1-\vartheta} \mathrm{e}^{-b(\vartheta-1) t}\right) u_{t}(q)^{\vartheta} \\
& =-b\left((\vartheta-1) c-u_{t}(q)\right) u_{t}(q)^{\vartheta} .
\end{aligned}
$$

Thus, we see that $u_{t}(q)$ solves

$$
\frac{\partial u_{t}(q)}{\partial t}=-\widetilde{\Psi}_{\vartheta}\left(u_{t}(q)\right), \quad u_{0}(q)=q,
$$

and, as in this partial differential equation, the function $\widetilde{\Psi}_{\vartheta}$ does not depend on $t$, which ensures that $\widetilde{Y}$ has in fact the time-homogeneous branching property. More precisely, $\widetilde{Y}$ is a CSBP with branching mechanism $\widetilde{\Psi}_{\vartheta}$.

\section{Acknowledgements}

J. Bertoin acknowledges support from ARCUS and FONDAP in Applied Mathematics that enabled his visit to CMM-DIM at Santiago during which the present joint work was undertaken. J. Fontbona thanks Nucleus Millennium ICM P04-069-F and FONDAP in Applied Mathematics for their support. S. Martínez thanks Nucleus Millennium ICM P04-069-F for their support. 


\section{References}

[1] Athreya, K. B. And Ney, P. E. (1972). Branching Processes. Springer, Berlin.

[2] Bertoin, J. ANd Le Gall, J.-F. (2000). The Bolthausen-Sznitman coalescent and the genealogy of continuousstate branching processes. Prob. Theory Relat. Fields 117, 249-266.

[3] Duquesne, T. and Le Gall, J.-F. (2002). Random trees, Lévy processes and spatial branching processes. Astérisque 281, 147 pp.

[4] Duquesne, T. and Winkel, M. (2007). Growth of Lévy trees. Prob. Theory Relat. Fields 139, 313-371.

[5] Grey, D. R. (1974). Asymptotic behaviour of continuous time continuous state-space branching processes. J. Appl. Prob. 11, 669-677.

[6] Kyprianou, A. (2006). Introductory Lectures on Fluctuations of Lévy Processes with Applications. Springer, Berlin.

[7] LagerÅs, A. N. And Martin-LöF, A. (2006). Genealogy for supercritical branching processes. J. Appl. Prob. 43, 1066-1076.

[8] Le Gall, J.-F. (1999). Spatial Branching Processes, Random Snakes and Partial Differential Equations. Birkhäuser, Basel.

[9] Lyons, R. AND Peres, Y. (2008). Probability on trees and networks. Available at http://mypage.iu.edu/ rdlyons/prbtree/book.pdf.

[10] NeveU, J. (1992). A continuous-state branching process in relation with the GREM model of spin glass theory. Res. Rep. 267, École Polytechnique.

[11] O'Connell, N. (1993). Yule process approximation for the skeleton of a branching process. J. Appl. Prob. 30, 725-729.

[12] Silverstein, M. L. (1968). A new approach to local times. J. Math. Mech. 17, 1023-1054. 